\title{
Approach to diagnosis of metabolic diseases
}

\author{
Enid Gilbert-Barness ${ }^{\mathrm{a}, \mathrm{b}}$ and Philip M. Farrell ${ }^{\mathrm{c}, \mathrm{d}, *}$ \\ a Department of Pathology, Cell Biology, Pediatrics and Obstetrics and Gynecology, Tampa General \\ Hospital, University of South Florida Morsani College of Medicine, Tampa, FL, USA \\ ${ }^{\mathrm{b}}$ Pathology and Laboratory Medicine, Distinguished Medical Alumni Professor Emeritus, University \\ of Wisconsin School of Medicine and Public Health, Madison, WI, USA \\ ${ }^{\mathrm{c}}$ Department of Pediatrics and Population Health Sciences, University of Wisconsin School of Medicine \\ and Public Health, Madison, WI, USA \\ ${ }^{\mathrm{d}}$ Department of Pediatrics, University of South Florida Morsani College of Medicine, Tampa General \\ Hospital, Tampa, FL, USA
}

\begin{abstract}
Inborn errors of metabolism are generally categorized as rare diseases. Their presentations are often so subtle and insidious as to cause daunting diagnostic challenges for even the most astute clinicians. Thus, irreversible morbidity and preventable mortality have been unavoidable until recent decades because of delayed diagnoses. This unfortunate circumstance has led to newborn screening programs worldwide for 40 or more hereditary metabolic disorders beginning with the dramatic improvements for patients with phenylketonuria in the 1960's. Increasingly sophisticated testing procedures such as tandem mass spectrometry and other multiplex technologies applied to dried blood spot specimens are now having greater impact without raising costs significantly. The advent of next generation sequencing methods is likely to stimulate further progress and lead to whole genome or exome sequencing as prenatal and neonatal screening expands further. With early diagnosis through screening and expedited therapies better outcomes are routinely possible, and even preventive therapies amounting to "cures" can be anticipated through research.
\end{abstract}

Keywords: Gene, genetic mutations, inborn errors of metabolism, hyperglycemia

\section{Conceptual overview}

The term "inborn errors of metabolism” was first used by Sir Archibald Garrod in his Croonian Lectures in 1908 [1] and in his monograph Inborn Errors of Metabolism in 1909 [2]. He defined these inborn errors as genetically determined diseases caused by blocks in the metabolic pathways due to deficient activity of an enzyme [3]. From Sir Garrod's observations of patients with alkaptonuria, albinism, cystinuria, and pentosuria, he developed the concept that certain diseases of lifelong duration arise because an enzyme governing a single metabolic step is reduced in activity or missing altogether [4]. Most of these disorders may be classified as rare diseases because newborn screening data show clearly that their incidence ranges from 1:4,000 for congenital hypothyroidism to $>1: 250,000$ (many disorders such as Maple Syrup Urine Disease (branched chain ketoaciduria).

\footnotetext{
*Corresponding author: Philip M. Farrell, Departments of Pediatrics and Population Health Sciences, UW School of Medicine and Public Health, 600 Highland Ave, Madison, WI 53792, USA. Tel: +1 608345 2308; Fax: +1 608 263 0510; E-mail: pmfarrell@wisc.edu.
} 
The definition of an inborn error of metabolism is arbitrary. Scriver et al. [5] include several hundred diseases with definite biochemical genetic bases. McKusick's catalog [6] contains several thousand diseases and disease states whose genetic abnormalities are described or assumed. Scriver et al. [5] solved the problem of exclusivity by adding "molecular" before encompassing every disease of the "textbook of the future." The 2nd Edition of Metabolic Diseases: Foundations of Clinical Management, Genetics, and Pathology [7] is limited to those diseases with either: 1) recognized biochemical abnormalities supplemented with pathologic and genetic information, and in which a mutation leads to an enzyme deficiency that causes a metabolic disease such as phenylketonuria (PKU); or 2) disorders associated with known genetic mutations that have altered cellular physiology such as the chloride channel defect in cystic fibrosis or caused severe structural, cellular, or subcellular abnormalities. Emphasis in the book has been given to the more common and/or well defined hereditary biochemical/metabolic disorders. Prototype conditions such as Lesch Nyhan Disease, an excellent example of a nucleotide (purine) metabolism disorder, are also emphasized.

Investigation of the molecular functions of genes has determined that they control the cellular metabolic function in the pathogenesis of disease far beyond that commonly understood as a metabolic disease. The genetics of variant human phenotypes is discussed by Scriver et al. [5] and Champion [8]. The relationship between gene and enzyme was described as "one gene, one enzyme" by Beadle and Tatum [9-11]. Tatum expanded this concept as follows: 1) all biochemical processes in all organisms are under genetic control; 2) these biochemical processes are resolvable into series of individual stepwise reactions; 3) each biochemical reaction is under the ultimate control of a different single gene; and 4) mutation of a single gene results only in an alteration in the ability of the cell to carry out a single primary chemical reaction.

Pauling et al. [12] discovered direct evidence that human mutations actually produce an alteration in the primary structure of proteins. Their seminal research revealed a valine substitution for glutamic acid in sickle cell hemoglobin. Ingram [13] showed that inborn errors of metabolism were caused by mutant genes that produced abnormal proteins whose functional activities were altered.

It is estimated that some 30,000 genes constitute the human genome. A variety of genome maps are available, including some that are disease specific such as the "cancer cell map" [14]. The genome map is being constantly improved, with updated gene sequences and locations of intronic elements, revised lists of benign SNPs, additional reference genomes, databases of variant frequencies that are ethnicityspecific, and associations of specific genes with specific human diseases. In addition, combinations of genes can result in a metabolic error. Thus, although $\sim 7000$ hereditary metabolic disorders are currently known, at least the same number of significant genetic errors as the number of genes seems theoretically possible, and an undetermined but large percentage of these may cause biochemical disorders with clinical impact. An estimation of genetic disease frequencies is shown in Table 1.

Table 1

Estimation of genetic disease frequencies

- $1 \%$ of live-born infants have monogenic disease

- $5 \%$ of individuals under 25 can expect to have a disease due to a genetic component

- $6 \%-8 \%$ of hospitalized children have monogenic disease

- $29 \%-41 \%$ of hospitalized children have gene-influenced disease

- $60 \%$ of older individuals have genetically influenced diseases, if multifactorial diseases are included

- $100 \%$ of humans carry 6-8 lethal genes

- $20 \%-30 \%$ of all infant deaths are due to genetic disorders

- $30 \%-50 \%$ of post-neonatal deaths are due to congenital malformations

- $50 \%$ of mental retardation has a genetic basis 


\section{Diagnostic strategies and tactics}

Clinical diagnosis of metabolic disease is made by specific tests, biochemical analyses, and histologic and genetic studies that are discussed by Leonard et al. [15] and Cleary et al. [16]. New methods such as next generation sequencing of genes $[17,18]$ and even whole genome sequencing $[19,20]$ are facilitating diagnoses and other goals such as genetic counseling. Clinicians seek diagnosis, effective treatment, an understanding of prognosis, and the capability to explain and counsel patients and their families. Parents and patients appreciate gaps in knowledge but may be left seriously disappointed or angry without a cure. Fortunately, our increased genetic knowledge and better understanding of molecular pathophysiology of the past two decades has led to dramatic improvements in therapies using novel methods such as small molecule modulators of defective proteins [e.g., CFTR modulators for cystic fibrosis [21], gene therapy [22], and stem cell infusions [23]]. Thus, investments in expedited diagnostic strategies such as newborn screening can pay great dividends when well targeted, highly effective therapies are available.

Identification of inborn errors of metabolism provides the basis for diagnosis, prognosis, genetic counseling, and, in an increasing number of patients, targeted treatments aimed directly at the fundamental defect. Many infants and children present as medical emergencies and life-preserving treatment is begun before a definite diagnosis is available. Diagnosis should be made rapidly and the implications and complexities of treatment explained in detail. Metabolic disease is usually not suspected in patients until more common diseases are considered. Those children with a history of consanguinity or those in families with a history of unexplained death, multiple spontaneous abortions, or previously diagnosed metabolic diseases have an increased likelihood of a metabolic disease. Frequently, children (and some adults) with metabolic diseases are first seen as critically ill patients with non-specific findings. Dehydration, acidosis, vomiting, ammonemia, hypoglycemia, or seizures must be managed aggressively. Inborn metabolic errors may be suspected if response to emergency treatment is not as expected.

Metabolic diseases can be divided into 3 main categories as listed in (Table 2). The majority of these disorders are inherited as autosomal recessive traits. Some such as Duchenne muscular dystrophy are X-linked. A few are inherited as dominant traits, and mitochondrial disorders form a genetically separate category. Mitochondrial enzymes are coded by both the maternal nuclear genome and by the mitochondrial DNA.

Clinical findings associated with metabolic diseases are listed in Table 3. It has been well recognized that symptoms may be quite variable, even in a group of patients with the same mutation. Thus, it has become clear that genetic modifiers may alter the expression of signs/symptoms and that gene-environment and nutrition-metabolism relationships can also influence the disease liability of pathologic genetic mutations.

The newborn with a severe metabolic abnormality may present with symptoms of apparent sepsis or asphyxia. These symptoms usually consist of irritability, failure to feed or suck, flaccidity, or coma. Previous miscarriages or sudden unexpected death in a sibling should trigger a screening investigation for metabolic disease. Sophisticated newborn screening using 4-5 blood spots on filter paper ["Guthrie cards" named for Robert Guthrie, the originator of population-based newborn screening with dried blood spots [24]], combined with tandem mass spectrometry as discussed by Ziadeh et al. [25] and Millington et al. [26] or molecular analyses [21] can identify a number of life-threatening diseases with a single, multiplex assay. The hereditary metabolic diseases that can now be screened for from filter paper blood spots in the newborn are listed in Table 4. Recently, severe combined immunodeficiency (SCID) has been added in many regions as well as "point of care" screening methods such as oxygen saturation measurements for critical congenital heart diseases.

Metabolic abnormalities may be suspected in infants with hydrops fetalis Table 3, or with placental abnormalities. Routine placental examination may disclose fetal metabolic storage disease by the 
Table 2

Categories of metabolic disease (Examples)

Large Complex Molecule Diseases

Complex lipid degradation (Gaucher, Niemann-Pick, Tay-Sachs, metachromatic leukodystrophy)

Glycoprotein degradation (fucosidase and mannosidase deficiency)

Mucopolysaccharidoses (Sanfilippo and Hurler syndrome)

Lipofuscin storage disorders (Batten disease)

Glycogen storage disease type I (von Gierke disease), type II (Pompe disease), type III (debrancher deficiency)

Peroxisomal disorders (Zellweger syndrome)

\section{Small Molecule Diseases}

Amino acid metabolism disorders (phenylketonuria, maple syrup urine disease, homocystinuria, tyrosinemia)

Ammonia metabolism disorders (ornithine transcarbamylase deficiency)

Organic acid metabolism disorders (methylmalonic acidemia, propionic acidemia, isovaleric acidemia)

Monosaccharides (galactosemia, hereditary fructose intolerance)

Pyruvic and lactic acid metabolism disorders (primary lactic acidosis, mitochondrial disorders)

Fatty acid metabolism disorders (medium-chain acyl-CoA dehydrogenase deficiency)

Purines and pyrimidine metabolism disorders (Lesch-Nyhan syndrome)

Vitamin and cofactor metabolism disorders (biotinidase deficiency)

Other Diseases

Metal metabolism disorders (Wilson and Menkes disease, hemochromatosis)

Lipoprotein metabolism disorders (Abetalipoproteinemia, familial hypercholesterolemia)

Porphyrin metabolism disorders (porphyria)

Membrane transport disorders (cystinuria, cystinosis)

Intestinal disaccharidases

Collagen and connective tissue disorders (Ehlers-Danlos disease, Marfan syndrome)

Congenital adrenal hyperplasia

Hyperinsulinism

presence of vacuolations of syncytiotrophoblasts, intermediate trophoblasts, and stromal Hofbauer cells. Suspected metabolic errors in the newborn are emergencies and require prompt identification and treatment. After obtaining the appropriate specimens for testing, emergency treatment must be instituted. If the patient dies, regardless of age, preparation should be made for a metabolic disease autopsy as discussed by Ernst et al. [27].

Pregnancy may occur in patients with metabolic diseases. Some of these conditions can be teratogenic. Many such pregnancies can be successfully managed with treatment. Treatment of these disorders is discussed in each chapter.

Examples of inborn errors of metabolism potentially occurring in high frequency among specific ethnic groups are listed in Table 5. However, it should be noted that some of these disorders are quite rare now in the era of prenatal diagnosis. For instance, the incidence of Tay Sachs disease in targeted Jewish populations is extremely low worldwide [28, 29].

The most common presentations of inborn errors are without gross physical anomalies and include disorders of amino acid, fatty acid, organic acid, and carbohydrate metabolism Table 6. Others present with dysmorphic features, neonatal deaths, self-mutilation, abnormal body or urine odor, hypotonia, deafness, or recurrent acidosis with or without ketosis. Failure to recognize and, when possible, treat these infants can result in irreversible neurologic damage.

Diagnostic strategy consists of meticulous attention to history and physical examination followed by appropriate screening tests, including biochemistry, radiology, or other modalities, and specific enzyme, protein, or gene analysis. Special educational services for children with inborn errors of metabolism may be available and therefore accuracy of diagnosis is essential as discussed by Powell et al. [30]. 
Table 3

Clinical findings associated with specific metabolic diseases

\begin{tabular}{|c|c|}
\hline Abdomen & Electron transport chain abnormalities \\
\hline Hepatomegaly & Disorders of fatty acid oxidation \\
\hline$\overline{\text { Galactosemia }}$ & Mucopolysaccharidoses \\
\hline Glycogen storage disease, type 1 & Myocardial infarction \\
\hline Hereditary fructose intolerance & Familial \\
\hline Fructose-1,6-diphosphatase deficiency & Hypocholesterolemia \\
\hline Methylmalonic acidemia & Fabry disease \\
\hline Propionic acidemia & Homocystinuria \\
\hline Glutaric acidemia, type II & Menkes disease \\
\hline \multicolumn{2}{|l|}{ Very-long-chain acyl-CoA deficiency } \\
\hline Medium-chain acyl-CoA deficiency & Diarrhea \\
\hline Short-chain acyl-CoA deficiency & Lysinuric protein intolerance \\
\hline Long-chain 3-OH acyl-CoA, dehydrogenase deficiency & Shwachman syndrome \\
\hline Carnitine transporter defect & Johansson Blizzard syndrome \\
\hline Carnitine palmityl transferase I o II deficiency & Pearson syndrome \\
\hline Acylcarnitine translocase deficiency & Congenital chloride diarrhea \\
\hline 3-hydroxy-3-methylglutaryl-CoA lyase deficiency & Glucose galactose malabsorption \\
\hline Phosphoenolpyruvate carboxykinase deficiency & Lactase deficiency \\
\hline Mitochondrial respiratory/electron transport chain defects & Sucrase deficiency \\
\hline Hereditary tyrosinemia, type I & Abetalipoproteinemia \\
\hline Argininosuccinicaciduria & $\underline{\text { Wolman disease }}$ \\
\hline$\alpha_{1}$-Antitrypsin deficiency & Diarrhea (Persistent) \\
\hline Smith-Lemli-Opitz syndrome & Sucrase isomaltase deficiency \\
\hline Zellweger syndrome & Acrodermatitis enteropathica \\
\hline Neonatal adrenoleukodystrophy & Congenital folate malabsorption \\
\hline \multicolumn{2}{|l|}{ Nieman-Pick disease, type C } \\
\hline Hepatosplenomegaly & Dysmorphic Features \\
\hline$\overline{\mathrm{GM}_{1} \text { gangliosidosis }}$ & Glutaric aciduria, type II \\
\hline I-cell disease & Glomerulopathy \\
\hline Gaucher disease, type I & Renal cystic dysplasia \\
\hline Niemann-Pick disease, type A, C & Cerebral dysgenesis \\
\hline Galactosialidosis & Facial dysmorphism \\
\hline Sialidosis & Congenital heart disease \\
\hline Mucopolysaccharidosis, type VII & Genital anomalies \\
\hline Wolman disease & Pyruvate dehydrogenase deficiency \\
\hline$\underline{\text { Pancreatitis }}$ & Microcephaly \\
\hline Glycogen storage disease, type I & "Fetal alcohol" facies \\
\hline Lipoprotein lipase deficiency & Agenesis of corpus callosum \\
\hline Homocystinuria & $\underline{\text { Peroxisomal Disorders }}$ \\
\hline Hydroxymethylgiutaryl-CoA lyase deficiency & Zellweger Syndrome \\
\hline Methylmalonic acidemia & Renal microcysts \\
\hline Propionic acidemia & Epiphyseal calcification \\
\hline \multirow[t]{3}{*}{ Pearson syndrome } & Facial dysmorphism \\
\hline & Congenital heart disease \\
\hline & Cerebral dysgenesis \\
\hline Chest & Hepatopathy \\
\hline Cardiomyopathy & $\underline{\text { Infantile Refsum disease }}$ \\
\hline Glycogen storage disease, type I & Facial dysmorphism \\
\hline Glycogen storage disease, type II & Hepatopathy \\
\hline Glycogen storage disease, type III & Rhizomelic chondrodysplasia punctata \\
\hline
\end{tabular}


Table 3

(Continued)

Facial dysmorphism

Rhizomelic limb shortening

$\mathrm{GM}_{1}$ gangliosidosis

Frontal bossing, low-set ears

Congenital adrenal hyperplasia

Ambiguous genitalia

$\underline{\text { Sialidosis }}$

Coarse facial features, stippled epiphyses

Mucolipidosis II

Coarse facial features

Mucopolysaccharidoses

Coarse facial features

Infantile sialic acid storage disease

Coarse facial features

$\beta$-Hydroxyisobutyryl-CoA deacylase deficiency

Congenital heart defects

Agenesis of corpus callosum

Dysmorphic facies

Gaucher-like storage disease

Arthrogryposis

Muscle phosphorylase deficiency

Arthrogryposis

Smith-Lemli-Opitz syndrome

Genital and digital abnormalities

Ears

Otic atrophy

Pyruvate dehydrogenase com plex deficiency

Leigh disease

Zellweger syndrome

\section{Extremities}

Arthritis

Alkaptonuria

Gaucher disease, type I

Lesch-Nyhan disease

Farber disease

I-cell disease

Mucolipidosis III

Homocystinuria

Mucopolysaccharidosis IS, HS

Bone or limb deformity

Dyostosis Multiplex

Hurler, Hurler-Scheie disease

Hunter disease

Sanfilippo disease

GM1 gangliosidosis

Mucolipidosis II, I-cell disease
Mucolipidosis III

Galactosialidosis

Maroteaux-Lamy disease

Sly disease

Multiple sulfatase deficiency

Osteoporosis and/or fractures

Propionic acidemia

Methylmalonic acidemia

Glycogen storage disease, type I

Homocystinuria

Adenosine deaminase deficiency

Lysinuric protein intolerance

Menkes disease

Infantile Refsum disease

Gaucher disease

I-cell disease

Eyes

Cataract

Galactosemia

Lowe syndrome

Mitochondrial respiratory electron transport chain defects

Zellweger syndrome

Rhizomelic chondrodysplasia punctata

Mevalonic aciduria

Corneal Clouding

I-cell disease

Steroid sulfatase deficiency

Dislocated lens

Methionine synthetase deficiency

Sulfite oxidase deficiency

Macular cherry-red spot

$\mathrm{GM}_{1}$ gangliosidosis

Galactosialidosis

Niemann-Pick Disease, type A

Tay-Sachs disease $\left(\mathrm{GM}_{2}\right.$ gangliosidosis)

Retinitis pigmentosa

Mitochondrial respiratory electron transport chain defects

Methylmalonic acidemia/Homocystinuria

Sjögren-Larsson syndrome

Zellweger syndrome

Neonatal adrenoleukodystrophy

Infantile Refsum disease

Abetalipoproteinemia

Long-chain 3-OH acyl-CoA dehydrogenase deficiency

\section{Head}

Alopecia 
Table 3

(Continued)

Multiple carboxylase deficiency

Cerebral calcification

Adrenoleukodystrophy

Abnormalities of folate metabolism

L-2-Hydroxyglutaric aciduria

Biopterin abnormalities

Coarse facial features

$\mathrm{GM}_{1}$ gangliosidosis

I-cell disease

Mucopolysaccharidosis, type VII

Sialidosis

Galactosialidosis

Macrocephaly

4-Hydroxybutyric aciduria

Glutaric aciduria, type I

L-2-Hydroxyglutaric aciduria

Neonatal adrenoleukodystrophy

Tay-Sachs disease

Hurler disease

Krabbe disease

Multiple sulfatase deficiency

Canavan disease

Mannosidosis

3-Hydroxy-3-methylglutaric aciduria

Pyruvate carboxylase deficiency

Multiple acyl-CoA dehydrogenase deficiency

Microcephaly

Short-chain Acyl-CoA dehydrogenase deficiency

Mitochondrial respiratory electron transport chain defects

Leigh disease

Steely hair

Menkes disease

\section{Hematology}

Hematologic abnormalities

Leukopenia with or without thrombopenia and anemia

Methylmalonic acidemia

Propionic acidemia

Isovaleric acidemia

3-Oxothiolase deficiency

Abnormalities of folate metabolism

Transcobalamin II deficiency

Shwachman syndrome

Pearson syndrome

Johansson Blizzard syndrome

Megaloblastosis

Cobalamin metabolic errors:

Methylmalonic acidemia

Homocystinuria

Transcobalamin II deficiency
Orotic aciduria

Mevalonic aciduria

Pearson syndrome

Abnormalities of folate metabolism

Shwachman syndrome

Johansson Blizzard syndrome

\section{Hydrops fetalis}

Gaucher disease

$\mathrm{GM}_{1}$ gangliosidosis

Salla disease

Sialidosis

Wolman disease

$\beta$-Glucuronidase deficiency

Congenital disorders of protein glycosylation

Deficiencies of red cell glycolytic/pentose phosphate pathway enzymes (eg,

G6PDH deficiency, private

kinase deficiency)

Farber disease

Fumarase deficiency

GSD IV

MPS VII (Sly disease)

MPS IVA (Morquio type A)

Mucolipidosis I (Sialidosis)

Mucolipidosis II (I-cell disease)

Neonatal hemochromatosis

Niemann-Pick C

Primary carnitine deficiency

Respiratory chain disorders

Sialic acid storage disease

Morquio syndrome

Neuraminidase deficiency

Myotonic dystrophy

Perinatal iron storage syndrome

Carnitine deficiency

Niemann-Pick type C

I-cell disease

Farber disease

Mucopolysaccharidosis type VII

Carbohydrate-deficient glycoprotein storage disease

\section{Mouth and throat}

Macroglossia

$\mathrm{GM}_{1}$ gangliosidosis

Macroglossia 
Table 3

(Continued)

\section{Neurological}

MLD, Niemann-Pick A and B, and Gaucher, type III

Gaucher disease, type II

Glutaric acidemia, type I

Krabbe disease

Crigler-Najjar syndrome

Phenylketonuria caused by a biopterin defect

Stroke-like episodes

Carbamoyl phosphate synthetase

MELAS

Carbohydrate deficient glycoprotein storage disease

Ethylmalonic aciduria

Fabry disease

Glutaric aciduria, type I

Homocystinuria

Isovaleric acidemia

Menkes disease

3-MTHF reductase deficiency

Methylmalonic acidemia

Ornithine transcarbamylase deficiency

Propionic acidemia

Purine nucleoside phosphorylase deficiency

Odor

Sweaty feet, strong

Glutaric aciduria (type II)

Isovaleric acidemia

Mousy or musty

Phenylketonuria

Maple syrup

Maple syrup urine disease

Tomcat urine

$\beta$-Methylcrotonylglycinuria

Cabbage

Methionine malabsorption

Rotting fish

Trimethylaminuria

Rancid fishy or cabbage

Tyrosinemia

Hoplike

Oasthouse disease

Swimming pool

Hawkinsinuria

\section{Psychiatric}

Sanfilippo syndrome (MPS III)
Hunter syndrome (MPS II)

X-linked ALD

Late-onset MLD

Late-onset GM2 gangliosidosis

Lesch-Nyhan syndrome

Porphyria

Wilson disease

Cerebrotendinous xanthomatosis

UCED

Homocystinuria due to MTHF reductase deficiency Adult-onset NCL

\section{Skin}

Angiokeratoma

Fabry disease

$\mathrm{GM}_{1}$ gangliosidosis

Fucosidosis

Galactosialidosis

Sialidosis

Desquamating, eczematous, or vesiculobullous lesions

Acrodermatitis enteropathica

Multiple carboxylase deficiency

Methylmalonic acidemia

Propionic acidemia

Hepatoerythropoietic porphyria

Congenital erythropoietic porphyria

Ichthyosis

Multiple sulfatase deficiency

$\mathrm{X}$-linked ichthyosis (steroid sulfatase deficiency)

Gaucher disease

Krabbe disease

Refsum disease

Sjögren-Larsson syndrome

Nodules

Farber disease (Ceramidase deficiency)

Thick skin

I-cell disease

$\mathrm{GM}_{2}$ gangliosidosis

Mucopolysaccharidosis, type VII

Sialidosis

Galactosialidosis

Xanthomas

Familial hypercholesterolemia

Lipoprotein lipase deficiency

Niemann-pick disease

ALD, adrenoleukodystrophy; MLD, metachromatic leukodystrophy; UCED, urea cycle enzyme defects; MTHF, methylenetetrahydrofolate; NCL, neuronal ceroid lipofuscinosis; MELAS, myoclonic epilepsy, lactic acidosis, and stroke. 
Table 4

Newborn screening panel: Core panel and secondary targets*

\begin{tabular}{|c|c|c|c|c|}
\hline \multirow[b]{2}{*}{ Acylcarnitines } & \multicolumn{4}{|l|}{ MS/MS } \\
\hline & & Amino acids & & \\
\hline \multirow{3}{*}{$\begin{array}{l}9 \text { Organic } \\
\text { Acidemia Disorders }\end{array}$} & 5 Fatty Acid & 6 Aminoacidopathies & 3 Hemoglobinopathies & 7 Other Genetic \\
\hline & Oxidation Defects & & & Disorders \\
\hline & & Core panel & & \\
\hline Isovaleric academia & $\begin{array}{l}\text { Medium-chain acyl-COA } \\
\text { dehydrogenase deficiency }\end{array}$ & Phenlketonuria & $\begin{array}{l}\text { Sickle cell anemia } \\
\text { (hemoglobin SS disease)* }^{*}\end{array}$ & Congenital Hypothyroidism \\
\hline Glutaric acidemia type I & Very long-chain acyl-CoA & Maple syrup disease & & Biotinidase deficiency \\
\hline $\begin{array}{l}\text { 3-hydroxy-3-methyl glutaric } \\
\text { aciduria (HMG) }\end{array}$ & dehydrogenase deficiency & $\begin{array}{l}\text { Homocystinuria* } \\
\text { (due to cystathionine beta }\end{array}$ & $\begin{array}{l}\text { Hemoglobin } \\
\text { S/ } \beta \text {-thalassemia* }\end{array}$ & $\begin{array}{l}\text { Congenital adrenal } \\
\text { hyperplasia* }\end{array}$ \\
\hline Multiple carboxylase deficiency & Long-chain L-3-hydroxy & synthase deficiency) & Hemoglobin S/C disease* & (21-hydroxylase deficiency) \\
\hline $\begin{array}{l}\text { Methylmalonic acidemia } \\
\text { (mutase deficiency)* }^{*}\end{array}$ & $\begin{array}{l}\text { acyl-CoA dehydrogenase } \\
\text { deficiency }\end{array}$ & Citrullinemia & & Classical galactosemia \\
\hline 3-Methylcrotonyl-CoA & & Argininosuccinic academia & & Hearing loss \\
\hline carboxylase deficiency* & $\begin{array}{l}\text { Trifunctional protein } \\
\text { deficiency }\end{array}$ & Tyrosinemia type $\mathrm{I}^{*}$ & & Cystic fibrosis \\
\hline $\begin{array}{l}\text { Methylmalonic acidemia } \\
\quad(\text { cobalamin disorders A \& B)* }\end{array}$ & Carnitine uptake defect & & & $\begin{array}{l}\text { Severe combined } \\
\text { immunodeficiency }\end{array}$ \\
\hline \multicolumn{5}{|l|}{ Propionic acidemia } \\
\hline \multicolumn{5}{|l|}{ B-Ketothiolase deficiency } \\
\hline & & Secondary targets & & \\
\hline 6 More Organic & 8 More Fatty Acid & 8 More & Other & 2 Other Hereditary \\
\hline Acidemia Disorders & Oxidation Defects & Aminioacidopathies & Hemoglobinopathies & Metabolic Disorders \\
\hline \multirow[t]{2}{*}{$\begin{array}{l}\text { Methylmalonic academia* } \\
\text { (cobalamin disorders C \& D) }\end{array}$} & $\begin{array}{l}\text { Short-chain acyl-CoA } \\
\text { dehydrogenase deficiency }\end{array}$ & $\begin{array}{l}\text { Benign } \\
\text { hyperphenylalaninemia }\end{array}$ & $\begin{array}{l}\text { Variant hemoglobinopathies* } \\
\text { (including hemoglobin E) }\end{array}$ & Galactokinase deficiency* \\
\hline & Glutaric acidemia type II & Tyrosinemia type II & & Galactose epimerase deficiency \\
\hline Malonic academia & $\begin{array}{l}\text { Medium/short-chain } \\
\text { L-3-hydroxy acyl-CoA } \\
\text { dehydrogenase deficiency }\end{array}$ & $\begin{array}{l}\text { Defects of biopterin cofactor } \\
\text { biosynthesis }\end{array}$ & & \\
\hline
\end{tabular}


Table 4

(Continued)

\begin{tabular}{|c|c|c|c|c|}
\hline & & Secondary targets & & \\
\hline 6 More Organic & 8 More Fatty Acid & 8 More & Other & 2 Other Hereditary \\
\hline Acidemia Disorders & Oxidation Defects & Aminioacidopathies & Hemoglobinopathies & Metabolic Disorders \\
\hline $\begin{array}{l}\text { Isobutyryl-CoA dehydrogenase } \\
\text { deficiency }\end{array}$ & $\begin{array}{l}\text { Medium-chain ketoacyl-CoA } \\
\text { thiolase deficiency }\end{array}$ & Argininemia & & \\
\hline $\begin{array}{l}\text { 2-Methyl 3-hydroxy butyric } \\
\text { aciduria }\end{array}$ & $\begin{array}{l}\text { Carnitine } \\
\text { palmitoyltransferase II } \\
\text { deficiency }\end{array}$ & Tyrosinemia type III & & \\
\hline $\begin{array}{l}\text { 2-Methylbutyryl-CoA } \\
\text { dehydrogenase deficiency }\end{array}$ & $\begin{array}{l}\text { Carnitine: acylcarnitine } \\
\text { translocase deficiency }\end{array}$ & $\begin{array}{l}\text { Defects of biopterin cofactor } \\
\text { regeneration }\end{array}$ & & \\
\hline \multirow[t]{2}{*}{ 3-Methylglutaconic aciduria } & $\begin{array}{l}\text { Carnitine } \\
\text { palmitoyltransferase I } \\
\text { deficiency (liver) }\end{array}$ & Hypermethioninemia & & \\
\hline & $\begin{array}{l}\text { Dienoyl-CoA reductase } \\
\text { deficiency }\end{array}$ & Citrullinemia type II & & \\
\hline
\end{tabular}

*From Newborn Screening: Toward a Uniform Screening Panel and System, Maternal and Child Health Bureau [Federal Register: March 8, 2005 (Volume 70, Number 44)]. [ http://mchb.hrsa.gov/screening/]. 
Table 5

Ethnic group incidence of inborn errors of metabolism

\begin{tabular}{|c|c|c|}
\hline Inborn Error & Ethnic Group & $\begin{array}{l}\text { Estimated Incidence } \\
\text { (per } 100000 \text { births) }\end{array}$ \\
\hline Hepatorenal tyrosinemia & $\begin{array}{l}\text { French-Canadians (Saguenay-Lac } \\
\text { Saint-Jean region) }\end{array}$ & $\begin{array}{l}54 \text { (before the introduction of } \\
\text { preventive prenatal diagnosis) }\end{array}$ \\
\hline Tay-Sachs disease & Canavan disease Ashkenazi Jews & $\begin{array}{l}33 \text { (before the introduction of } \\
\text { preventive prenatal diagnosis) }\end{array}$ \\
\hline Gaucher disease, type 1 & Canavan disease Ashkenazi Jews & 100 \\
\hline \multirow[t]{3}{*}{ Phenylketonuria (PKU) } & Canavan disease Ashkenazi Jews & 5 \\
\hline & Yemenite Jews & 19 \\
\hline & Turkish & 38.5 \\
\hline Porphyria variegata & South African (white) & 300 \\
\hline Congenital adrenal hyperplasia & Yupik Eskimos & 200 \\
\hline Glutaric aciduria, type 1 & Ojibway Indians (Canada) & $>50$ \\
\hline Maple syrup urine disease & Mennonites (Pennsylvania) & 568 \\
\hline
\end{tabular}

From: Clarke JTR, (Ed.): A Clinical Guide to Inherited Metabolic Diseases - 3rd ed., Cambridge University Press, 2006.

Clinical investigation is augmented by a large number of common blood and urine tests (blood, sugar, urine $\mathrm{pH}$, anion gap, cytopenia, liver function tests, sweat test, electroencephalogram, and radiography of bones). Radiologic examination may include X-rays, CT scans, and MR imaging. MRI scans should be substituted for CT scans whenever possible to avoid radiation exposure. Ophthalmologic and neurophysiologic investigations are often important. Laboratory findings in inborn errors of metabolism are listed in Table 7. Abnormal metabolites are shown in Table 8.

Abnormalities in serum electrolytes can be caused by vomiting or diarrhea. Inclusion of bicarbonate and $\mathrm{pH}$ assessment may indicate a large anion gap due to the presence of organic acids. Electrolytes, however, may be normal with organic acidemias.

Neutropenia or pancytopenia suggests the presence of one of the organic acidemias. A urinalysis may indicate the presence of reducing substances and the presence or absence of ketones. Ketonuria is not found in normal newborns. Urine $\mathrm{pH}$ above 5.0 in an acidotic infant may suggest renal tubular acidosis. Absence of ketones in association with hypoglycemia may indicate an error in fatty acid metabolism. It is helpful to save a small amount of frozen urine and plasma for later evaluation of keto acids, carnitine, and organic acids.

Elevated serum ammonia levels are found in patients with liver disease and in those with errors of the urea cycle and several of the organic acidemias. Transient hyperammonemia occurs in some newborns, and this is reversible when treatment is prompt. Urea cycle errors are frequently associated with very low blood urea levels. Some metabolic disorders cause liver cirrhosis in infants and children and some liver disorders progress to stupor and coma.

Liver function tests commonly indicate primary liver diseases. However, abnormal liver function test findings result from many of the metabolic diseases, including disorders of organic and amino acid metabolism, and many of the storage diseases.

Elevated serum lactate is usually due to hypoxemia or poor perfusion and occurs with sepsis. It may be due, however, to improper technique in drawing blood without good blood flow. Lactic acid is produced in the anaerobic metabolism of glucose through pyruvate, which is then converted in the liver to glucose. Deficiency of pyruvate dehydrogenase complex is the most common error of metabolism that causes lactic acidosis. 
Table 6

Laboratory findings in some inborn errors of metabolism

\begin{tabular}{|c|c|}
\hline Lactic acidosis \pm & Hyperammonemia \\
\hline Increased anion gap & Ornithine transcarbamylase \\
\hline Methylmalonic acidemia & deficiency \\
\hline Propionic acidemia & Carbamoyl phosphate \\
\hline Isovaleric acidemia & synthetase deficiency \\
\hline Multiple carboxylase deficiency & Argininosuccini- \\
\hline Maple syrup urine disease & caciduria \\
\hline Glutaric acidemia, type I & Citrullinemia \\
\hline Glutaric acidemia, type II & Methylmalonic acidemia \\
\hline Short-chain acyl-CoA & Propionic acidemia \\
\hline dehydrogenase deficiency & Isovaleric acidemia \\
\hline Long-chain 3-OH acyl-CoA & Multiple carboxylase deficiency \\
\hline dehydrogenase deficiency & Glutaric acidemia, type II \\
\hline Ketothiolase deficiency & Very-long-chain acyl-CoA \\
\hline Acetoacetate CoA ligase deficiency & dehydrogenase deficiency \\
\hline 3-hydroxy-3-methylglutaryl & Medium-chain acyl-CoA \\
\hline CoA Iyase deficiency & dehydrogenase deficiency \\
\hline $\begin{array}{l}\text { Pyruvate dehydrogenase } \\
\text { complex deficiency }\end{array}$ & $\begin{array}{l}\text { Short-chain acyl-CoA dehydrogenase } \\
\text { deficiency }\end{array}$ \\
\hline $\begin{array}{l}\text { Pyruvate carboxylase } \\
\text { deficiency }\end{array}$ & $\begin{array}{l}\text { Acylcarnitine translocase } \\
\text { deficiency }\end{array}$ \\
\hline Phosphoenolpyruvate & Ketosis \\
\hline carboxykinase deficiency & Methylmalonic acidemia \\
\hline Mitochondrial respiratory/electron & Propionic acidemia \\
\hline transport chain defects & Isovaleric acidemia \\
\hline Leigh disease & Multiple carboxylase deficiency \\
\hline Hereditary galactosemia & Maple syrup urine disease \\
\hline Glycogen storage disease, & Glutaric acidemia, type II \\
\hline type I & Short-chain acyl-CoA dehydrogenase \\
\hline Hereditary fructose intolerance & deficiency \\
\hline Fructose-1,6-diphosphatase & Ketothiolase deficiency \\
\hline deficiency & Acetoacetate-CoA ligase \\
\hline Hereditary tyrosinemia, & deficiency \\
\hline type I & Pyruvate carboxylase deficiency \\
\hline Respiratory alkalosis & Glycogen storage disease, type I \\
\hline $\begin{array}{l}\text { Omithine transcarbamylase } \\
\text { deficiency }\end{array}$ & \\
\hline $\begin{array}{l}\text { Carbamoyl phosphate synthetase } \\
\text { deficiency }\end{array}$ & \\
\hline Argininosuccinicaciduria & \\
\hline Citrullinemia & \\
\hline
\end{tabular}

Fructose-1,6-diphosphatase deficiency

Hypoglycemia

Hyperinsulinism

Glycogen storage disease, type I

Hereditary fructose intolerance

Fructose-1,6-diphosphatase deficiency

Glutaric acidemia, type I

Glutaric acidemia, type II

Very-long-chain acyl-CoA dehydrogenase deficiency

Medium-chain acyl-CoA dehydrogenase deficiency

Short-chain acyl-CoA dehydrogenase deficiency

Long-chain 3-OH acyl-CoA dehydrogenase deficiency

Carnitine transporter defect

Carnitine palmitoyl transferase I deficiency

Carnitine palmitoyl transferase II deficiency

Acylcarnitine translocase deficiency

Ketothiolase deficiency

Acetoacetate-CoA ligase deficiency

3-hydroxy-3-methylglutaryl-CoA

Iyase deficiency

Hereditary galactosemia

Neonatal hemochromatosis

Mitochondrial respiratory/electron transport chain defects

\section{Lipemia}

Glycogen storage disease, type I

Myopathy can be caused by a few of the glycogen storage diseases or mitochondrial disorders. Muscle biopsy or fibroblast cultures may be diagnostic. Myoglobinuria may be present, and serum free carnitine may be decreased.

Peroxisomal action is responsible for $\beta$-oxidation of fatty acids, and deficiency results in developmental retardation. Peroxisomal disorders with such manifestations include Zellweger syndrome, 
Table 7

Laboratory evaluation for suspected inborn errors of metabolism

Comments

\author{
Initial Evaluation* \\ Blood tests \\ CBC with differential \\ Blood glucose \\ Electrolytes, BUN, creatinine, uric acid \\ Arterial blood gas \\ Serum ammonia
}

AST, ALT, bilirubin, PT

$\mathrm{LDH}$, aldolase, creatine, kinase

Urine Tests

Color, odor

Urinalysis

Reducing substances

Myoglobin

Specialized Tests

Blood tests

Quantitative plasma amino acids

Lactate and pyruvate

Acylcarnitine profile

\section{Urine Tests}

Qualitative urine organic acids
Should be obtained from artery or vein without a tourniquet; the tube should be placed on ice for transport to the laboratory and analyzed immediately. If the plasma ammonia concentration is $>100 \mathrm{micromol} / \mathrm{L}(1.7 \mathrm{mcg} / \mathrm{mL})$, the measurement should be repeated immediately

If the patient has signs or symptoms of myopathy

If the patient has signs or symptoms of myopathy

Plasma amino acid analysis must be performed quantitatively rather than qualitatively

Lactate and pyruvate should be measured in arterial blood that is, transported on ice

Analysis of acylcarnitine conjugates is performed by tandem mass spectrometry and can be measured in a plasma sample or a filter-paper bloodspot; serum is preferred because of inherent problems in quantitating compounds from a filter-paper blood spot

Minimum of 2 to $5 \mathrm{~mL}$ in sterile container without preservative

CBC: complete blood count; ALT: alanine aminotransferase; BUN: blood urea nitrogen; PT: prothrombin time; AST: aspartate aminotransferase; LDH: lactate dehydrogenase. * If possible, blood and urine samples should be obtained for both the initial and specialized tests at the time of presentation. Samples for specialized tests should be processed and stored appropriately for further testing if indicated.

adrenoleukodystrophy, and oxalosis [31]. Very-long-chain fatty acids are elevated in the serum with most forms of peroxisomal disorders.

Many chemical screening programs such as determination of organic and amino acids, oligosaccharides, and glycosaminoglycans in blood and urine are available for diagnostic workup of suspected progressive degenerative metabolic diseases. Distinguishing biochemical findings of inborn errors of metabolism are shown in Table 9.

Some inherited metabolic diseases significantly increase the risk of intercurrent illness. For example, recurrent treatment-resistant otitis media is a common problem in children with mucopolysaccharide storage diseases because distortion of the Eustachian tube and production of particularly tenacious 
Table 8

Abnormal metabolites in metabolic disorders

\begin{tabular}{|c|c|}
\hline Disorder & Abnormal Metabolite (s)* \\
\hline \multicolumn{2}{|l|}{ Organic Acidemias } \\
\hline Methylmalonic academia & Methylmalonic and methylcitric acids \\
\hline Propionic academia & $\begin{array}{l}\text { 3-Hydroxpropionic acid propionylglycine, methylcitric } \\
\text { acid }\end{array}$ \\
\hline Isovaleric academia & Isovalerylglycine \\
\hline Glutaric academia type I & Glutaric and 3-hydroxyglutaric acids \\
\hline 3-Methylglutaconic aciduria & 3-Methylglutaconic acid \\
\hline $\begin{array}{l}\text { 2-Hydroxy-3-methylbutyryl-CoA dehydrogenase } \\
\text { deficiency }\end{array}$ & $\begin{array}{l}\text { 2-Hydroxy-3-methylblutyric acid, tiglyglycine, } \\
\text { 2-methyl-3-hydroxyacetoacetic acid }\end{array}$ \\
\hline \multicolumn{2}{|l|}{ Disorders of Ketogenesis } \\
\hline $\begin{array}{l}\text { Medium chain acyl-CoA dehydrogenase (MCAD) } \\
\text { deficiency }\end{array}$ & Hexanoylglycine and suberylglycine \\
\hline 3-ketothiolase deficiency & $\begin{array}{l}\text { 2-hydroxy-3-methylbutyric acid, tiglyglycine, } \\
\text { 2-methyl-3-hydroxyacetoacetic acid }\end{array}$ \\
\hline $\begin{array}{l}\text { 3-Hydroxy-3-methylglutaryl (HMG)-CoA lyase } \\
\text { deficiency }\end{array}$ & $\begin{array}{l}\text { 3-Hydroxy-3-methylglutaric, 3-methylglutaric and } \\
\text { 3-methylglutaconic acids }\end{array}$ \\
\hline \multicolumn{2}{|l|}{ Other Disorders } \\
\hline Canavan disease & $\mathrm{N}$-acetylaspartic acid \\
\hline $\begin{array}{l}\text { Glutaric academia type II (multiple acyl-CoA } \\
\text { dehydrogenase deficiency) }\end{array}$ & Glutaric and 2-hydroxyglutaric acids \\
\hline Mevalonate kinase deficiency & Mevalonic acid \\
\hline Maple syrup urine disease & 2-Hydroxyisovaleric and 2-hydroxy-3-methylvaleric \\
\hline Phenylketonuria & Phenylpyruvic and phenyllactic acids \\
\hline Fumarase deficiency & Fumaric acid \\
\hline Glutathione synthetase deificneyc & 5-Oxoproline (pyroglutamic acid) \\
\hline Biotinidase deficiency* & $\begin{array}{l}\text { 3-Hydroxyisovaleric acid, methylcitric acid, } \\
\text { 3-methylcrotonlglycine, propionylglycine }\end{array}$ \\
\hline Holocarboxylase synthetase deficiency* & $\begin{array}{l}\text { 3-Hydroxyisovaleric acid, methylcitric acid, } \\
\text { 3-methylcrotonylglycine, propionylglycine }\end{array}$ \\
\hline Glycerol kinase deficiency & Glycerol \\
\hline $\begin{array}{l}\text { Ethylmalonic encephalopathy } \\
\text { Disorders of Uncertain Consequence }\end{array}$ & Ethylmalonic and methylsuccinic acids \\
\hline 3-methylcrotonyl-CoA carboxlase deficiency & 3-methylcrotonylglycine \\
\hline Short chain acyl-CoA dehydrogenase deficiency & Ethylmalonic acid, butyrylglycine \\
\hline
\end{tabular}

*Other abnormal metabolites may be seen in these disorders but those listed are the most characteristic for the diagnosis.

mucus combine to create a favorable environment for bacterial colonization of the middle ear. The neutropenia that is a prominent feature of glycogen storage disease type $\mathrm{Ib}$, and some of the organic acidopathies, predisposes to pyogenic infections. Classic galactosemia predisposes infants to neonatal Escherichia coli sepsis [32].

Major congenital malformations, such as meningomyelocele, complex congenital heart disease, and major congenital limb deformities, are not generally considered signs of an underlying inherited metabolic disease; however, some inherited metabolic conditions occur with dysmorphism so characteristic that a strong presumptive diagnosis can be made on physical examination alone [32]. A large 
Table 9

Distinguishing biochemical findings of inborn errors of metabolism

\begin{tabular}{|c|c|c|c|c|c|c|c|c|}
\hline Findings & MSUD & $\mathrm{OA}$ & $\mathrm{UCD}$ & DCM & FAO & $\mathrm{MD}$ & PD & LSD \\
\hline Metabolic acidosis & \pm & ++ & - & \pm & \pm & \pm & - & - \\
\hline Respiratory alkalosis & - & - & + & - & - & - & - & - \\
\hline Hyperammonemia & \pm & + & ++ & - & \pm & - & - & - \\
\hline Hypoglyclemia & \pm & \pm & - & + & + & \pm & - & - \\
\hline Ketones & $\mathrm{A} / \mathrm{H}$ & $\mathrm{H}$ & A & $\mathrm{A} / \mathrm{H}$ & $\mathrm{A} / \mathrm{L}$ & $\mathrm{A} / \mathrm{H}$ & A & A \\
\hline Lactic acidosis & \pm & \pm & - & + & \pm & ++ & - & - \\
\hline
\end{tabular}

MSUD: maple syrup urine disease; OA: organic acidemias' UCD: urea cycle disorders; DCM: disorders of carbohydrate metabolism; FAO: fatty acid oxidation disorders; MD: mitochondrial disorders; PD: peroxisomal disorders; LSD: lysosomal storage disorder. -: usually absent; \pm : sometimes present; +: usually present; ++: always present. A: appropriate; H: inappropriately high; L: inappropriately low. Adapted from: Weiner DL, Metabolic Emergencies. In: Textbook of Pediatric Emergency Medicine, 5th ed. Fleisher GR, Ludwig S, Henretig FM (Eds). Lippincott, Williams \& Wilkins, Philadelphia, 2006.

number of metabolic diseases present with gross abnormalities of appearance Table 10A and 10B. Many of these have lysosomal defects, and most will have hepatosplenomegaly. These include such diseases as mucopolysaccharidoses, sphingolipidoses, and other lysosomal storage diseases. These diseases are not acutely life-threatening. Children with these conditions commonly have developmental delay. Many appear normal at birth and progressively deteriorate Table 3.

An important step between the clinical findings and the biochemical findings is the anatomic pathology, including histology, histochemistry, immunochemistry, and electron microscopy (EM). For example, screening peripheral blood for cytoplasmic vacuoles in the lymphocytes as shown in Fig. 1 may be the first indication of a storage disorder.

Some metabolic disorders present as acute encephalopathy. The earliest signs of encephalopathy may be no more obvious than excessive drowsiness, unusual behavior, or some unsteadiness of gait. Acute or intermittent ataxia is a common sign of acute encephalopathy in older children with inborn errors of metabolism. A history of recurrent attacks of unsteadiness of gait or ataxia, especially when associated with vomiting or deterioration of consciousness, should stimulate investigation of a possible inherited metabolic disease Table 11 [25].

In addition to the biochemical tests of blood and urine, solid tissues and cell cultures may be needed for biochemical assays. A biopsy specimen should be divided for biochemical analysis and morphologic investigation. This is particularly important in liver, muscle, and intestinal biopsies and in skin biopsies made for the fibroblast cultures.

Hypoglycemia occurs with starvation in young infants but also may indicate an error in gluconeogenesis, errors in fat metabolism and defects in hormone metabolism. A rapid response to glucose, especially in the presence of hepatomegaly, may suggest glycogen storage disease or fructose-1,6diphosphatase deficiency. An approach to diagnosis when a patient presents with hypoglycemia is shown in Fig. 2. This algorithm will guide clinicians toward a proper sequence of diagnostic tests.

Even after death, some of the techniques used in the living can be helpful in detecting inborn errors of metabolism. For example, even small amounts of urine may remain in the bladder and should be aspirated and saved. Tissues and body fluids should be obtained and maintained in a state suitable for the tests desired. New diseases and diagnostic tests are identified at a surprisingly rapid rate. Very few laboratories perform all the tests. 
Table 10A

Physical examination findings as clues to inborn errors of Metabolism

\begin{tabular}{|c|c|}
\hline Finding & Potential Inborn Error \\
\hline \multicolumn{2}{|l|}{ General } \\
\hline $\begin{array}{l}\text { Tall, long-limbed body habitus } \\
\text { Head }\end{array}$ & Homocysturia \\
\hline $\begin{array}{l}\text { Coarse facial features (eg, hirsuitism } \\
\text { prominent brow ridge, and gingival } \\
\text { hypertrophy) }\end{array}$ & $\begin{array}{l}\text { Oligosaccharidoses, mucopolysaccharidoses, } \\
\text { mucolipidoses }\end{array}$ \\
\hline Microcephaly & $\begin{array}{l}\text { Untreated phenylketonuria (PKU), maternal PKU syndrome, } \\
\text { congenital disorders of protein glycosylation, } \\
\text { leukodystrophies (late, organic acidemias, urea cycle } \\
\text { disorders, maple syrup urine disease }\end{array}$ \\
\hline Macrocephaly & $\begin{array}{l}\text { Canavan disease, glutaric academia type I, oligosaccharidoses, } \\
\text { mucopolysaccharidoses, mucolipidoses, Tay-Sachs (early) }\end{array}$ \\
\hline \multicolumn{2}{|l|}{ Hair } \\
\hline Alopecia & Biotinidase deficiency, vitamin D resistant rickets \\
\hline Sparse & Biotinidase deficiency, Menkes disease \\
\hline Kinky, brittle & $\begin{array}{l}\text { Argininosuccinic aciduria and citrullinemia (due to arginine } \\
\text { deficiency), Menkes disease, mucopolysaccharidoses }\end{array}$ \\
\hline \multicolumn{2}{|l|}{ Eyes } \\
\hline Cataracts & $\begin{array}{l}\text { Oligosaccharidoses, Fabry diseiase, neuronal ceroid } \\
\text { lipofuscinosis, galactosemia, Smith-Lemli-Opitz syndrome } \\
\text { peroxisome bioigenesis defects, rhizomelic chondrodysplasia } \\
\text { punctata, Wilson disease, errors of mitochondrial oxidative } \\
\text { phosphorylation }\end{array}$ \\
\hline Cherry red spot & $\begin{array}{l}\text { Tay-Sachs disease, Sandhoff disease, Sialidosis type I and type } \\
\text { II, GM1-gangliosidosis, Niemann-Pick disease type A, } \\
\text { Gaucher disease type 2, metachromatic leukodystrophy, } \\
\text { galactosialidosis }\end{array}$ \\
\hline Corneal clouding & $\begin{array}{l}\text { Oligosaccharidoses, mucopolysaccharidoses, mucolipidoses, } \\
\text { Tangier, sialidosis }\end{array}$ \\
\hline Corneal opacity & $\begin{array}{l}\text { Oligosaccharidoses, Fabry disease, steroid sulfatase deficiency } \\
\text { (X-linked ichthyosis), Tangier, molybdenum cofactor } \\
\text { deficiency, sulfite oxidase deficiency }\end{array}$ \\
\hline Dislocated lens & Homocystinuria, sulfite oxidase deficiency \\
\hline Kayser-Fleischer rings & Wilson disease \\
\hline Retitinitis pigmentosa & $\begin{array}{l}\text { Abetalipoproteinemia, peroxisome biogenesis disorders of } \\
\text { protein glycosylation, fatty acid oxidation defects, } \\
\text { mitochondrial defects, mucopolysaccharidoses, Krabbe } \\
\text { disease, Menkes disease, disorders of cobalamin (vitamin } \\
\text { B12) transport and synthesis, ornithine aminotransferase } \\
\text { deficiency }\end{array}$ \\
\hline
\end{tabular}

Adapted from Wappner Rs, Hainline BE. Inborn errors of metabolism. In: Oski's Pediatrics, Principles and Practice, 3rd ed., McMillan JA, DeAngelis CD, Feigin RD, Warshaw JB (Eds), Lippincott, Williams and Wilkins, Philadelphia, 1999. Saudubray JM, Chappentier C. Clinical phenotypes: Diagnosis/algorithms. In: Metabolic and Molecular Bases of Inherited Disease, 8th ed, Scriver CR, Beaudet AL, Sly WS, Valle D (Eds), McGrall-Hill, New York, 2001.Lindor NM, Karnes PS. Initial assessment of infants and children with suspected inborn errors of metabolism. Mayo Clin Proc 70:987, 1995. Cleary MA, Green A. Developmental delay: when to suspect and how to investigate for an inborn error of metabolism, Arch Dis Child 90:1128, 2005. 
Table 10B

Physical examination findings as clues to inborn errors of Metabolism

\begin{tabular}{ll}
\hline Finding & Potential Inborn Error \\
\hline Hearing loss & $\begin{array}{c}\text { Peroxisomal disorders, mitochondrial disorders, lysosomal storage } \\
\text { disorders, mucopolysaccharidoses }\end{array}$ \\
$\begin{array}{l}\text { Mouth } \\
\text { Gingival hyperplasia } \\
\text { Chest }\end{array}$ & Oligosaccharidoses, mucopolysaccharidoses, mucolipidoses \\
$\begin{array}{l}\text { Inverted nipples } \\
\text { Abdomen } \\
\text { Hepatosplenomegaly } \\
\text { Hepatomegaly }\end{array}$ & $\begin{array}{l}\text { Congenital disorders of protein glycosylation } \\
\end{array}$ \\
& $\begin{array}{l}\text { Lysosomal storage disease } \\
\text { Glycogen storage diseases, carnitine palmitoyltransferase II deficiency } \\
\text { (infantile form), peroxisomal disorders, mitochondrial DNA depletion } \\
\text { disorders, tyrosinemia type I (hepatorenal), mucolipidoses, congenital } \\
\text { disorders of protein glycosylation, longer chain fatty acid oxidation }\end{array}$ \\
disorders
\end{tabular}

\section{Musculoskeletal}

Arthritis

Neurologic

Dystonia

Myopathy

Paresthesia

Peripheral neuropathy

Psychoses

\section{Skin}

Hypopigmentation or absent pigment

Angiokeratoma

Dermatitis

Edema

Hirsutism

Ichthyosis

Photosentization

Xanthomas
Farber disease, purine metabolism disorders

Glutaric acidemia I, organic acidemias, Wilson disease, mitochondrial disorders

Fatty acid oxidation defects, mitochondrial disorders, Pompe disease and other glycogen storage diseases

Fabry disease, sialidosis

Congenital disorders of protein glycosylation, leukodystrophies, peroxisomal disorders, Tangier disease

Adult MLD Tay-Sachs, homocystinuria, porphyrias, purine metabolism disorders

Cystinosis, Menkes disease, phenylketonuria, sialidosis

Fabry, fucosidosis, galactosialidosis, beta-mannosidosis, sialidosis

Biotinidase deficiency, Hartup disease, phenylketonuria, prolidase deficiency

GM1 gangliosidosis, prolidase deficiency

Oligosaccharidoses, mucopolysaccharidoses, mucolipidoses

Multiple sulfatase deficiency, isolated steroid sulfatase deficiency

Porphyrias

Hyperlipoproteinemias and other disorders of lipoproteins

Adapted from Wappner Rs, Hainline BE. Inborn errors of metabolism. In: Oski's Pediatrics, Principles and Practice, 3rd ed., McMillan JA, DeAngelis CD, Feigin RD, Warshaw JB (Eds), Lippincott, Williams and Wilkins, Philadelphia, 1999. Saudubray JM, Chappentier C. Clinical phenotypes: Diagnosis/algorithms. In: Metabolic and Molecular Bases of Inherited Disease, 8th ed, Scriver CR, Beaudet AL, Sly WS, Valle D (Eds), McGrall-Hill, New York, 2001.Lindor NM, Karnes PS. Initial assessment of infants and children with suspected inborn errors of metabolism. Mayo Clin Proc 70:987, 1995. Cleary MA, Green A. Developmental delay: when to suspect and how to investigate for an inborn error of metabolism, Arch Dis Child 90:1128, 2005. 


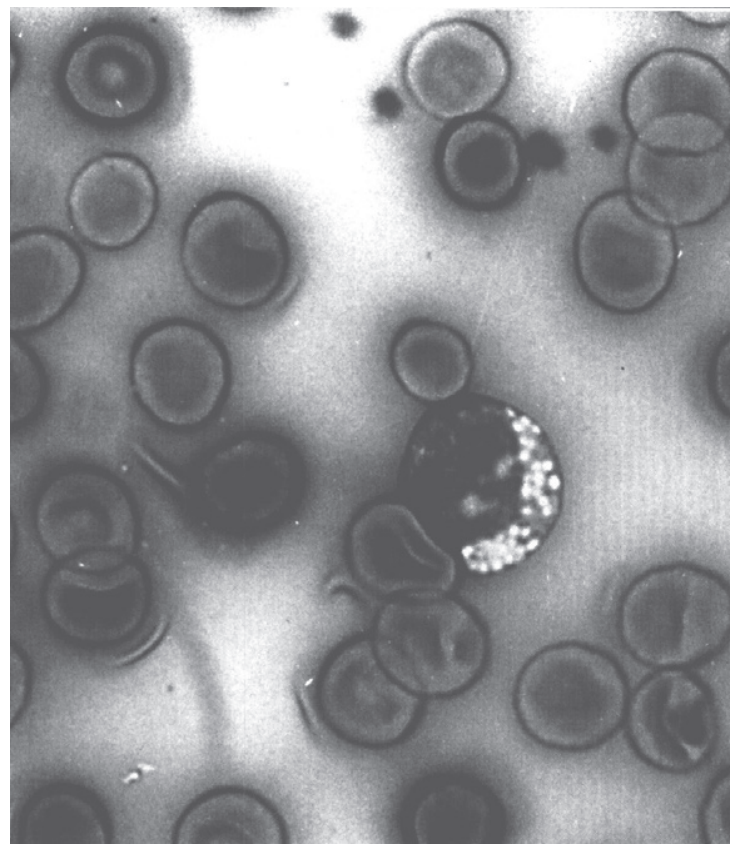

Fig. 1. Vacuolated lymphocyte in the peripheral blood of a patient with mannosidosis.

Table 11

Differential diagnosis of acute encephalopathy in metabolic diseases

\begin{tabular}{lccccc}
\hline & UCED & MSUD & OAuria & FAOD & ETC Defects \\
\hline Metabolic acidosis & 0 & \pm & +++ & \pm & ++ \\
Plasma glucose & $\mathrm{N}$ & $\mathrm{N}$ or $\downarrow$ & $\downarrow \downarrow$ & $\downarrow \downarrow$ & $\mathrm{N}$ \\
Urinary ketones & $\mathrm{N}$ & $\uparrow \uparrow$ & $\uparrow \uparrow$ & 0 & 0 \\
Plasma ammonia & $\uparrow \uparrow \uparrow$ & $\mathrm{N}$ & $\uparrow \uparrow$ & $\mathrm{N}$ & $+\uparrow \uparrow$ \\
Plasma lactate & $\mathrm{N}$ & $\mathrm{N}$ & $\mathrm{N}$ & $\mathrm{N}$ & $\mathrm{N}$ \\
Liver function & $\pm \mathrm{N}$ & $\mathrm{N}$ & $\downarrow \uparrow$ & $\downarrow \downarrow$ & \\
Plasma carnitine & $\mathrm{N}$ & $\mathrm{N}$ & $\uparrow$ glycine & \pm & $\mathrm{N}$ \\
Plasma amino acids & Abnormal & $\uparrow$ BCAA & Abnormal & Abnormal & $\mathrm{N}$ \\
Urinary organic acids & $\mathrm{N}$ & Abnormal & &
\end{tabular}

UCED, urea cycle enzyme defect; MSUD, maple syrup urine disease; OAuria, organic aciduria; FAOD, fatty acid oxidation defect; ETC, mitochondrial electron transport chain; BCAA, branched-chain amino acids; $\uparrow$, elevated; $\downarrow$ decreased;+, present; \pm , variably present; N, normal; O, not present. From: Clarke JTR, (Ed.): A Clinical Guide to Inherited Metabolic Diseases - 3rd ed., Cambridge University Press, 2006.

\section{Further development of prenatal and neonatal screening}

As techniques such as next generation sequencing $[17,18]$ and whole genome sequencing are developed further for routine use, there will be many more commercial laboratories offering comprehensive testing as has already proved helpful for newborn intensive care units $[19,20]$. However, reporting and ethical issues need to be resolved before whole genome sequencing can be used widely [33]. 


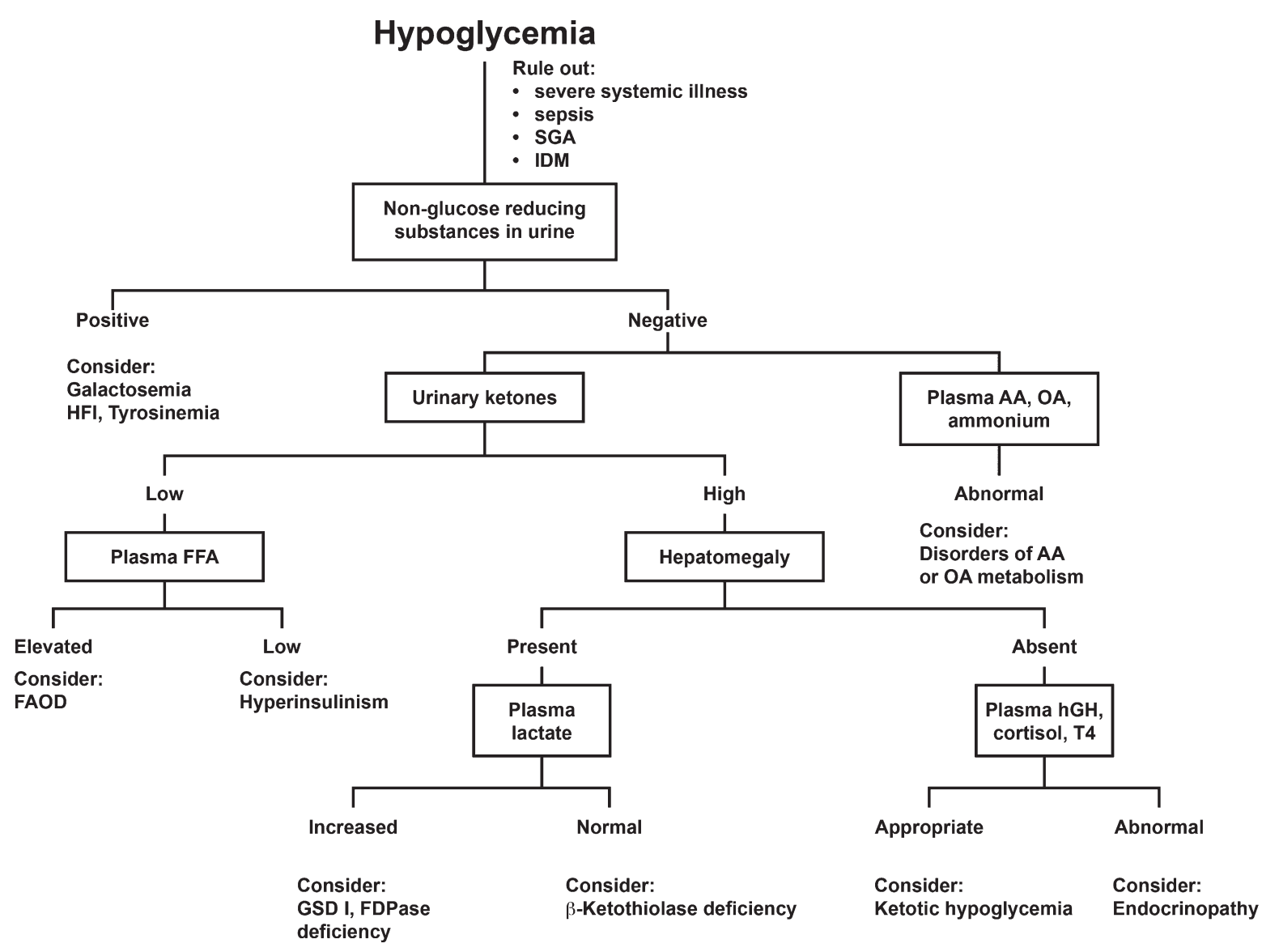

Fig. 2. Approach to the differential diagnosis of hypoglycemia. Abbreviations: SGA, small for gestational age; IDM, infant of diabetic mother; HFI, hereditary fructose intolerance; AA, amino acids; OA, organic acids; FFA, free fatty acids; FAOD, fatty acid oxidation defect; hGH, human growth hormone; T4, thyroxine; GSD, glycogen storage disease; FDPase, fructose6-diphosphatase. Clarke JTR, (Ed.): A Clinical Guide to Inherited Metabolic Diseases - 3rd ed., Cambridge University Press, 2006.

\section{References}

[1] A.E. Garrod, Inborn errors of metabolism (Croonian Lectures), Lancet 2 (1908), 1.

[2] A.E. Garrod, Inborn Errors of Metabolism, London, 1909, Oxford University Press.

[3] A.E. Garrod, Inborn Errors of Metabolism, London, 1923, Oxford University Press.

[4] A.G. Bearn and E.D. Miller, Archibald Garrod and the development of the concept of inborn errors of metabolism. Bull Hist Med 53 (1979), 315-325.

[5] C.R. Scriver, A.L. Beaudet, W.S. Sly and D. Valle, editors: The Metabolic and Molecular Bases of Inherited Disease, 7th ed, New York, 1995, McGraw-Hill.

[6] V.A. McKusick, Mendelian Inheritance in Man, 11th ed, Baltimore, 1994, The Johns Hopkins University Press.

[7] E. Gilbert-Barness, L. Barness and P. Farrell (Eds.), Metabolic Diseases: Foundations of Clinical Management, Genetics, and Pathology. IOS Press, Amsterdam, In press.

[8] M.O. Champion, An approach to the diagnosis of inherited metabolic disease. Arch Dis Child Educ Pract Ed 95 (2010), 40-46.

[9] G.W. Beadle, Biochemical genetics. Chem Rev 37 (1945), 15.

[10] G.W. Beadle and E.L. Tatum, Genetic control of biochemical reactions in Neurospora. Proc Natl Acad Sci USA 27 (1941), 499-506.

[11] E.L. Tatum, A case history of biological research. Science 129 (1959), 1711-1715. 
[12] L. Pauling, H.A. Itano, S.J. Singer and I.C. Wells, Sickle cell anemia: A molecular disease. Science 110 (1949), 543-548.

[13] V.M. Ingram, A specific chemical difference between the globins of normal human and sickle cell anaemia haemoglobin. Nature 178 (1956), 792-794.

[14] N.J. Krogan, S. Lippman, D.A. Agard, A. Ashworth and T. Ideker, The cancer cell map initiative: Defining the hallmark networks of cancer. Mol Cell $\mathbf{5 8}$ (2015), 690-698.

[15] J.V. Leonard and A.A. Morris, Diagnosis and early management of inborn errors of metabolism presenting around the time of birth. Acta Paediatr 95 (2006), 6-14.

[16] M.A. Cleary and A. Green, Developmental delay: When to suspect and how to investigate for an inborn error of metabolism. Arch Dis Child 90 (2005), 1128-1132.

[17] L. Francescatto and N. Katsanis. Newborn screening and the era of medical genomics. Semin Perinatol 39 (2015), 617-622.

[18] M.W. Baker, A.E. Atkins, S.K. Cordovado, M. Hendrix, M.C. Earley and P.M. Farrell, Improving newborn screening for cystic fibrosis using next-generation sequencing technology: A technical feasibility study. Genet Med 18(3) (2016), 231-238.

[19] C.J. Saunders, N.A. Miller, S.E. Soden, D.L. Dinwiddie, A. Noll, N.A. Alnadi, N. Andraws, M.L. Patterson, L.A. Krivohlavek, J. Fellis, S. Humphray, P. Saffrey, Z. Kingsbury J.C. Weir, J. Betley, R.J. Grocock, E.H. Margulies, E.G. Farrow, M. Artman, N.P. Safina, J.E. Petrikin, K.P. Hall and S.F. Kingsmore. Rapid whole-genome sequencing for genetic disease diagnosis in neonatal intensive care units. Sci Transl Med 4 (2012), 154ra135.

[20] E.A. Worthey, A.N. Mayer, G.D. Syverson, D. Helbling, B.B. Bonacci, B. Decker, J.M. Serpe, T. Dasu, M.R. Tschannen, R.L. Veith, M.J. Basehore, U. Broeckel, A. Tomita-Mitchell, M.J. Arca, J.T. Casper, D.A. Margolis, D.P. Bick, M.J. Hessner, J.M. Routes, J.W. Verbsky, H.J. Jacob and D.P. Dimmock, Making a definitive diagnosis: Successful clinical application of whole exome sequencing in a child with intractable inflammatory bowel disease. Genet Med 13 (2011), 255-262.

[21] M.D. Amaral, Novel personalized therapies for cystic fibrosis: Treating the basic defect in all patients. J Int Med 277 (2015), 155-166.

[22] S.J. Kolb and J.T. Kissel, Spinal muscular atrophy. Neurol Clin 33 (2015), 831-846.

[23] M.S. Falzarano, C. Scotton, C. Passarelli and A. Ferlini, Duchenne muscular dystrophy: From diagnosis to therapy. Molecules 20 (2015), 18168-18184.

[24] R. Guthrie and A. Susi. A simple phenylalanine method for detecting PKU in large populations of newborn infants. Pediatrics 32 (1963), 338-343.

[25] R. Ziadeh, E.P. Hoffman, D.N. Finegold, et al., Medium chain acyl-CoA dehydrogenase deficiency in Pennsylvania: Neonatal screening shows high incidence and unexpected mutation frequencies. Pediatr Res 37 (1995), 675-678.

[26] D.S. Millington, N. Kodo, D.L. Norwood and C.R. Roc, Tandem mass spectrometry: A new method for acylcarnitine profiling with potential for neonatal screening for inborn errors of metabolism. J Inherit Metab Dis 13 (1990), $321-324$.

[27] L.M. Ernst, N. Sondheimer, M.A. Deardorff, et al., The value of the metabolic autopsy in the pediatric hospital setting. J Pediatr 148 (2006), 779-783.

[28] M. Kaback, J. Lim-Steele, D. Dabholkar, D. Brown, N. Levy and K. Zeiger, Tay-Sachs disease-carrier screening, prenatal diagnosis, and the molecular era. An international perspective, 1970 to 1993. The International TSD Data Collection Network. JAMA 270 (1993), 2307-2315.

[29] R.M. Lew, L. Burnett, A.L. Proos, K. Barlow-Stewart, M.B. Delatycki, A. Bankier, H. Aizenberg, M.J. Field, Y. Berman, R. Fleischer and M. Fietz, Ashkenazi Jewish population screening for Tay-Sachs disease: The international and Australian experience. J Paediatr Child Health 51 (2015), 271-279.

[30] K. Powell, Van Naarden, K. Braun and R. Singh, et al., Prevalence of developmental disabilities and receipt of special education services among children with an inborn error of metabolism. J Pediatr 156 (2010), 420-426.

[31] N.E. Braverman, M.D. D’Agostino and G.E. Maclean. Peroxisome biogenesis disorders: Biological, clinical and pathophysiological perspectives. Dev Disabil Res Rev 17 (2013), 187-196.

[32] J.T.R. Clarke, (Ed.), A Clinical Guide to Inherited Metabolic Diseases - 3rd ed., Cambridge University Press, 2006.

[33] M.J. Deem, Whole-Genome Sequencing and Disability in the NICU: Exploring Practical and Ethical Challenges. Pediatrics 137(Suppl 1) (2016), S47-S55. 